\title{
ON A HYBRID VERSION OF THE VINOGRADOV MEAN VALUE THEOREM
}

\author{
CHANGHAO CHEN AND IGOR E. SHPARLINSKI
}

\begin{abstract}
Given a family $\varphi=\left(\varphi_{1}, \ldots, \varphi_{d}\right) \in \mathbb{Z}[T]^{d}$ of $d$ distinct nonconstant polynomials, a positive integer $k \leqslant d$ and a real
\end{abstract} positive parameter $\rho$, we consider the mean value

$$
M_{k, \rho}(\boldsymbol{\varphi}, N)=\int_{\mathbf{x} \in[0,1]^{k}} \sup _{\mathbf{y} \in[0,1]^{d-k}}\left|S_{\boldsymbol{\varphi}}(\mathbf{x}, \mathbf{y} ; N)\right|^{\rho} d \mathbf{x}
$$

of exponential sums

$$
S_{\boldsymbol{\varphi}}(\mathbf{x}, \mathbf{y} ; N)=\sum_{n=1}^{N} \exp \left(2 \pi i\left(\sum_{j=1}^{k} x_{j} \varphi_{j}(n)+\sum_{j=1}^{d-k} y_{j} \varphi_{k+j}(n)\right)\right),
$$

where $\mathbf{x}=\left(x_{1}, \ldots, x_{k}\right)$ and $\mathbf{y}=\left(y_{1}, \ldots, y_{d-k}\right)$. The case of polynomials $\varphi_{i}(T)=T^{i}, i=1, \ldots, d$ and $k=d$ corresponds to the classical Vinaogradov mean value theorem.

Here motivated by recent works of Wooley (2015) and the authors (2019) on bounds on $\sup _{\mathbf{y} \in[0,1]^{d-k}}\left|S_{\boldsymbol{\varphi}}(\mathbf{x}, \mathbf{y} ; N)\right|$ for almost all $\mathbf{x} \in[0,1]^{k}$, we obtain nontrivial bounds on $M_{k, \rho}(\varphi, N)$.

\section{IntRODUCTION}

For an integer $\nu \geqslant 2$, let $\mathrm{T}_{\nu}=(\mathbb{R} / \mathbb{Z})^{\nu}$ be the $\nu$-dimensional unit torus. We denote

$$
\mathbf{e}(x)=\exp (2 \pi i x)
$$

The exponential sums

$$
S_{d}(\mathbf{u} ; N)=\sum_{n=1}^{N} \mathbf{e}\left(u_{1} n+\ldots+u_{d} n^{d}\right), \quad \mathbf{u}=\left(u_{1}, \ldots, u_{d}\right) \in \mathrm{T}_{d},
$$

introduced and estimated by Weyl [9], are commonly called the Weyl sums.

Weyl sums appear in a great variety of number theoretic problems starting with the problem of uniformly of distribution of fractional parts of real polynomials, see also [1]. They also play a crucial role in estimating the zero-free region of the Riemann zeta-function and thus

2010 Mathematics Subject Classification. 11K38, 11L15.

Key words and phrases. Weyl sums, hybrid mean values, discrepancy. 
in turn in bounding in the error term in the prime number theorem, see [8, Section 8.5], and the Waring problem, see [8, Section 20.2], in estimating short character sums modulo highly composite numbers $[8$, Section 12.6].

Thanks to recent striking results of Bourgain, Demeter and Guth [3] (for $d \geqslant 4$ ) and Wooley [10] (for $d=3$ ) (see also [12]), for the mean value of $S_{d}(\mathbf{u} ; N)$ we have

$$
\int_{\mathrm{T}_{d}}\left|S_{d}(\mathbf{u} ; N)\right|^{2 s(d)} d \mathbf{u} \leqslant N^{s(d)+o(1)}, \quad N \rightarrow \infty
$$

where

$$
s(d)=\frac{d(d+1)}{2}
$$

which is the best possible form of the Vinogradov mean value theorem.

On the other hand, for individual sums it is known that their size depends on Diophantine properties of the coefficients $u_{1}, \ldots, u_{d}$, but generally the situation is not well understood, see $[4,5]$.

The following best known bound is a direct implication of (1.1) and is given in $\left[2\right.$, Theorem 5]. Let $\mathbf{u}=\left(u_{1}, \ldots, u_{d}\right) \in \mathrm{T}_{d}$ be such that for some $\nu$ with $2 \leqslant \nu \leqslant d$ and some positive integers $a$ and $q$ with $\operatorname{gcd}(a, q)=1$ we have

$$
\left|u_{\nu}-\frac{a}{q}\right| \leqslant \frac{1}{q^{2}}
$$

Then for any $\varepsilon>0$ there exits a constant $C(\varepsilon)$ such that

$$
\left|S_{d}(\mathbf{u} ; N)\right| \leqslant C(\varepsilon) N^{1+\varepsilon}\left(q^{-1}+N^{-1}+q N^{-\nu}\right)^{\frac{1}{d(d-1)}} .
$$

Recently, Wooley [11] has considered a hybrid scenario which interpolates between individual bounds and mean value estimates. In this settings one seeks results which hold for all values of the components of $\mathbf{u}=\left(u_{1}, \ldots, u_{d}\right) \in \mathrm{T}_{d}$ on some prescribed set of positions and almost all values of the components on the remaining positions.

Given a family $\varphi=\left(\varphi_{1}, \ldots, \varphi_{d}\right) \in \mathbb{Z}[T]^{d}$ of $d$ distinct nonconstant polynomials and a sequence of complex weights $\mathbf{a}=\left(a_{n}\right)_{n=1}^{\infty}$, for $\mathbf{u}=$ $\left(u_{1}, \ldots, u_{d}\right) \in \mathrm{T}_{d}$ we define the trigonometric polynomials

$$
T_{\mathbf{a}, \boldsymbol{\varphi}}(\mathbf{u} ; N)=\sum_{n=1}^{N} a_{n} \mathbf{e}\left(u_{1} \varphi_{1}(n)+\ldots+u_{d} \varphi_{d}(n)\right) .
$$

Furthermore, for $k=1, \ldots, d$, we decompose

$$
\mathrm{T}_{d}=\mathrm{T}_{k} \times \mathrm{T}_{d-k} .
$$


Given $\mathbf{x} \in \mathrm{T}_{k}, \mathbf{y} \in \mathrm{T}_{d-k}$ we refine the notation (1.3) and write

$$
T_{\mathbf{a}, \boldsymbol{\varphi}}(\mathbf{x}, \mathbf{y} ; N)=\sum_{n=1}^{N} a_{n} \mathbf{e}\left(\sum_{j=1}^{k} x_{j} \varphi_{j}(n)+\sum_{j=1}^{d-k} y_{j} \varphi_{k+j}(n)\right) .
$$

If $\mathbf{a}=\mathbf{e}=(1)_{n=1}^{\infty}$ (that is, $a_{n}=1$ for each $n \in \mathbb{N}$ ) we just write

$$
T_{\varphi}(\mathbf{x}, \mathbf{y} ; N)=T_{\mathbf{e}, \varphi}(\mathbf{x}, \mathbf{y} ; N) \text {. }
$$

In fact Wooley [11] has studied only the classical case $a_{n}=1$ for all $n \in \mathbb{N}$ and the polynomials

$$
\left\{\varphi_{1}(T), \ldots, \varphi_{d}(T)\right\}=\left\{T, \ldots, T^{d}\right\} .
$$

(we note that the order of $\varphi_{1}, \ldots, \varphi_{d}$ is not specified in (1.5)). Embedding in the argument of Wooley [11, Theorem 1.1] the modern form of the Vinogradov mean value theorem (1.1), one derives that for almost all $\mathbf{x} \in \mathrm{T}_{k}$ with respect to the $k$-dimensional Lebesgue measure on $\mathrm{T}_{k}$, we have

$$
\sup _{\mathbf{y} \in \mathrm{T}_{d-k}}\left|T_{\boldsymbol{\varphi}}(\mathbf{x}, \mathbf{y} ; N)\right| \leqslant N^{\left(1+\Delta_{W}(\boldsymbol{\varphi}, k)\right) / 2+o(1)}, \quad N \rightarrow \infty
$$

where

$$
\Delta_{W}(\boldsymbol{\varphi}, k)=\frac{2 \sigma_{k}(\boldsymbol{\varphi})+d-k+1}{2 s(d)+d-k+1}
$$

with $s(d)$ given by (1.2) and

$$
\sigma_{k}(\boldsymbol{\varphi})=\sum_{j=k+1}^{d} \operatorname{deg} \varphi_{j}
$$

The authors [6] have extended and improved this and some other results of Wooley [11]. In particular, by [6, Theorems 2.1, 2.3 and Corollary 2.4], the bound (1.6) holds with

$$
\Delta_{C S}(\boldsymbol{\varphi}, k)=\min \left\{\frac{2 \sigma_{k}(\boldsymbol{\varphi})+d-k}{2 s(d)+d-k}, \frac{\sigma_{k}(\boldsymbol{\varphi})+1}{s(d)}\right\}<\Delta_{W}(\boldsymbol{\varphi}, k)
$$

instead of $\Delta_{W}(\boldsymbol{\varphi}, k)$ and also applies to sums $T_{\mathbf{a}, \boldsymbol{\varphi}}(\mathbf{x}, \mathbf{y} ; N)$ with complex weights $a_{n}=n^{o(1)}, n \in \mathbb{N}$, and a large family of polynomials.

\section{HyBRID MEAN VALUE THEOREMS}

2.1. Notation and conventions. Throughout the paper, the notation $U=O(V), U \ll V$ and $V \gg U$ are equivalent to $|U| \leqslant c V$ for some positive constant $c$, which throughout the paper may depend on the degree $d$ and occasionally on the small real positive parameter $\varepsilon$.

For any quantity $V>1$ we write $U=V^{o(1)}$ (as $V \rightarrow \infty$ ) to indicate a function of $V$ which satisfies $|U| \leqslant V^{\varepsilon}$ for any $\varepsilon>0$, provided $V$ 
is large enough. One additional advantage of using $V^{o(1)}$ is that it absorbs $\log V$ and other similar quantities without changing the whole expression.

We use $\# \mathcal{S}$ to denote the cardinality of a finite set $\mathcal{S}$.

We say that some property holds for almost all $\mathbf{x} \in \mathrm{T}_{k}$ if it holds for a set $\mathcal{X} \subseteq \mathrm{T}_{k}$ of $k$-dimensional Lebesgue measure $\lambda(\mathcal{X})=1$.

2.2. Main results. Here, instead of asking about bounds on

$$
\sup _{\mathbf{y} \in \mathbf{T}_{d-k}}\left|T_{\mathbf{a}, \varphi}(\mathbf{x}, \mathbf{y} ; N)\right|,
$$

for a real $\rho>0$ we consider the mean value

$$
M_{k, \rho}(\mathbf{a}, \boldsymbol{\varphi}, N)=\int_{\mathbf{x} \in \mathbf{T}_{k}} \sup _{\mathbf{y} \in \mathrm{T}_{d-k}}\left|T_{\mathbf{a}, \boldsymbol{\varphi}}(\mathbf{x}, \mathbf{y} ; N)\right|^{\rho} d \mathbf{x}
$$

Let

$$
W(T ; \boldsymbol{\varphi})=\operatorname{det}\left(\varphi_{i}^{(j-1)}(T)\right)_{i, j=1}^{n}
$$

denote the Wronskian of $d$ polynomials $\varphi=\left(\varphi_{1}, \ldots, \varphi_{d}\right) \in \mathbb{Z}[T]^{d}$.

We recall that $s(d)$ and $\sigma_{k}(\boldsymbol{\varphi})$ are given by (1.2) and (1.7), respectively.

Theorem 2.1. Suppose that $\varphi \in \mathbb{Z}[T]^{d}$ is such that the Wronskian $W(T ; \boldsymbol{\varphi})$ does not vanish identically. Let $\mathbf{a}=\left(a_{n}\right)_{n=1}^{\infty}$ be a sequence of complex weights with $a_{n}=n^{o(1)}$. Then for any real positive $\rho \leqslant$ $2 s(d)+d-k$ we have

$$
M_{k, \rho}(\mathbf{a}, \boldsymbol{\varphi}, N) \leqslant N^{\mu(\boldsymbol{\varphi}, k) \rho+o(1)}, \quad N \rightarrow \infty
$$

where

$$
\mu(\boldsymbol{\varphi}, k)=\frac{s(d)+\sigma_{k}(\boldsymbol{\varphi})+d-k}{2 s(d)+d-k} .
$$

Note that Theorem 2.1 gives a non-trivial bound, that is, $\mu(\boldsymbol{\varphi}, k)<$ 1 , provided that $\sigma_{k}(\boldsymbol{\varphi})<s(d)$. Moreover for $k=d$, we have $\sigma_{d}(\boldsymbol{\varphi})=0$ and thus we recover the bound (1.1) in the Vinogradov mean value theorem.

From Theorem 2.1 we derive the following bounds for the mean values of short sums. For $K \in \mathbb{Z}$, we consider Weyl sums over short intervals

$$
S_{d}(\mathbf{u} ; K, N)=\sum_{n=K+1}^{K+N} \mathbf{e}\left(u_{1} n+\ldots+u_{d} n^{d}\right), \quad \mathbf{u}=\left(u_{1}, \ldots, u_{d}\right) \in \mathrm{T}_{d} .
$$

We now give an upper bound on the mean value of the largest value of all such sums, that is, for $\sup _{K \in \mathbb{Z}}\left|S_{d}(\mathbf{u} ; K, N)\right|$. No result of this type seems to be known prior to this work. 
Corollary 2.2. For any real positive $\rho \leqslant d^{2}+2 d-1$ we have

$$
\int_{\mathbf{T}_{d}} \sup _{K \in \mathbb{Z}}\left|S_{d}(\mathbf{u} ; K, N)\right|^{\rho} d \mathbf{u} \leqslant N^{\mu_{d} \rho+o(1)}, \quad N \rightarrow \infty,
$$

where

$$
\mu_{d}=1-\frac{d}{d^{2}+2 d-1}
$$

Recalling the definition of (1.3), (1.4), for $\mathbf{x} \in \mathrm{T}_{k}$ we can write

$$
\sup _{\mathbf{y} \in T_{d-k}}\left|T_{\mathbf{a}, \boldsymbol{\varphi}}(\mathbf{x}, \mathbf{y} ; N)\right|=\sup _{\mathbf{u} \in T_{d} \cap \pi_{d, k}^{-1}(\mathbf{x})}\left|T_{\mathbf{a}, \boldsymbol{\varphi}}(\mathbf{u} ; N)\right|,
$$

where $\pi_{d, k}$ is the orthogonal projection of $\mathrm{T}_{d}$ onto $\mathrm{T}_{k}$, that is,

$$
\pi_{d, k}:\left(u_{1}, \ldots, u_{d}\right) \rightarrow\left(u_{1}, \ldots, u_{k}\right) .
$$

This suggests that one can try to extend Theorem 2.1 to more general setting by taking some other mappings instead of the orthogonal projection $\pi_{d, k}$.

Now, more generally, given a mapping $f: \mathbb{R}^{d} \rightarrow \mathbb{R}^{k}$, for $\mathbf{x} \in \mathbb{R}^{k}$ we denote

$$
f^{-1}(\mathbf{x})=\left\{\mathbf{u} \in \mathbb{R}^{d}: f(\mathbf{u})=\mathbf{x}\right\} .
$$

Then for $\rho>0$ we define

$$
\mathrm{M}_{k, f, \rho}(\mathbf{a}, \boldsymbol{\varphi}, N)=\int_{\mathbb{R}^{k}} \sup _{\mathbf{u} \in \mathrm{T}_{d} \cap f^{-1}(\mathbf{x})}\left|T_{\mathbf{a}, \boldsymbol{\varphi}}(\mathbf{u} ; N)\right|^{\rho} d \mathbf{x},
$$

where $T_{\mathbf{a}, \varphi}(\mathbf{u} ; N)$ is given by $(1.3)$.

In the following we first take $f$ to be an orthogonal projection onto some $k$-dimensional subspace, and second we take $f$ to be some Hölder mapping.

Let $\mathcal{G}(d, k)$ denote the collections of all $k$-dimensional linear subspaces of $\mathbb{R}^{d}$. For $\mathcal{V} \in \mathcal{G}(d, k)$, let $\pi_{\mathcal{V}}: \mathbb{R}^{d} \rightarrow \mathcal{V}$ denote the orthogonal projection onto $\mathcal{V}$.

For the degree sequence $\operatorname{deg} \varphi_{1}, \ldots, \operatorname{deg} \varphi_{d}$ we denote them as

$$
r_{1} \leqslant \ldots \leqslant r_{d}
$$

and define

$$
\widetilde{\sigma}_{k}(\boldsymbol{\varphi})=\sum_{i=k+1}^{d} r_{i} .
$$

Theorem 2.3. Suppose that $\varphi \in \mathbb{Z}[T]^{d}$ is such that the Wronskian $W(T ; \boldsymbol{\varphi})$ does not vanish identically. Let $\mathbf{a}=\left(a_{n}\right)_{n=1}^{\infty}$ be a sequence 
of complex weights with $a_{n}=n^{o(1)}$. If $\mathcal{V} \in \mathcal{G}(d, k)$, then for any real positive $\rho \leqslant 2 s(d)+d-k$ we have

$$
\mathrm{M}_{k, \pi_{\mathcal{V}, \rho}}(\mathbf{a}, \boldsymbol{\varphi}, N) \leqslant N^{\mu_{\mathcal{V}}(\boldsymbol{\varphi}, k) \rho+o(1)}, \quad N \rightarrow \infty,
$$

where

$$
\mu_{\mathcal{V}}(\boldsymbol{\varphi}, k)=\frac{s(d)+\widetilde{\sigma}_{k}(\boldsymbol{\varphi})+d-k}{2 s(d)+d-k} .
$$

Now we turn to $\vartheta$-Hölder functions, for some $0<\vartheta \leqslant 1$, that is, functions $f: \mathbb{R}^{d} \rightarrow \mathbb{R}^{k}$ which satisfy

$$
\|f(\mathbf{x})-f(\mathbf{y})\| \ll\|\mathbf{x}-\mathbf{y}\|^{\vartheta},
$$

where $\|\mathbf{z}\|$ is the Euclidean norm of $\mathbf{z}$ (note that the left side of this inequality is the Euclidean norm in $\mathbb{R}^{k}$, while the right side is the Euclidean norm in $\mathbb{R}^{d}$ ). In particular, in the case $\vartheta=1$ the function $f$ is often called a Lipschitz function.

Theorem 2.4. Let $f: \mathbb{R}^{d} \rightarrow \mathbb{R}^{k}$ be a $\vartheta$-Hölder map for some $0<\vartheta \leqslant 1$. Let $\mathbf{a}=\left(a_{n}\right)_{n=1}^{\infty}$ be a sequence of complex weights with $a_{n}=n^{o(1)}$. Suppose that $\boldsymbol{\varphi} \in \mathbb{Z}[T]^{d}$ is such that the Wronskian $W(T ; \boldsymbol{\varphi})$ does not vanish identically. Denote

$$
\delta(\boldsymbol{\varphi})=\min _{i=1, \ldots, d} \operatorname{deg} \varphi_{i}
$$

Then for any real positive $\rho \leqslant 2 s(d)+d-k \vartheta$ we have

$$
\mathrm{M}_{k, f, \rho}(\mathbf{a}, \boldsymbol{\varphi}, N) \leqslant N^{\mu_{\vartheta}(\boldsymbol{\varphi}, k) \rho+o(1)}, \quad N \rightarrow \infty
$$

where

$$
\mu_{\vartheta}(\boldsymbol{\varphi}, k)=\frac{s(d)+\sigma_{0}(\boldsymbol{\varphi})+d-(\delta(\boldsymbol{\varphi})+1) \vartheta k}{2 s(d)+d-k \vartheta}
$$

Note that for the classical choice of polynomials (1.5), we have $\delta(\boldsymbol{\varphi})=1, \sigma_{0}(\boldsymbol{\varphi})=s(d)$ and thus

$$
\mu_{\vartheta}(\boldsymbol{\varphi}, k)=\frac{2 s(d)+d-2 k \vartheta}{2 s(d)+d-k \vartheta}<1
$$

for any $k>0$. Therefore Theorem 2.4 gives a non-trivial bound for $\mathrm{M}_{k, f, \rho}(\mathbf{a}, \boldsymbol{\varphi}, N)$ for any $k>0$ and any $\vartheta$-Hölder function $f$ with $0<$ $\vartheta \leqslant 1$. Moreover if $\vartheta \rightarrow 0$ then $\mu_{\vartheta}(\boldsymbol{\varphi}, k) \rightarrow 1$. Indeed, it is expected that if the function $f$ becomes "bad" ( $\vartheta$ becomes "small") then the bounds for $\mathrm{M}_{k, f, \rho}(\mathbf{a}, \boldsymbol{\varphi}, N)$ also become "bad". However, we do not know whether there exits a continuous function $f: \mathbb{R}^{d} \rightarrow \mathbb{R}^{k}$ such that we do not have non-trivial bounds for $\mathrm{M}_{k, f, \rho}(\mathbf{a}, \boldsymbol{\varphi}, N)$, that is, for any $\rho>0$ and any $\varepsilon>0$, one has

$$
\mathrm{M}_{k, f, \rho}(\mathbf{a}, \boldsymbol{\varphi}, N) \gg N^{1-\varepsilon}
$$


for infinitely many $N \in \mathbb{N}$.

2.3. Hybrid mean value theorems for discrepancy. Similar to works of Wooley [11] and the authors [6], we obtain similar results for the discrepancy.

Let $\xi_{n}, n \in \mathbb{N}$, be a sequence in $[0,1)$. The discrepancy of this sequence at length $N$ is defined as

$$
D_{N}=\sup _{0 \leqslant a<b \leqslant 1}\left|\#\left\{1 \leqslant n \leqslant N: \xi_{n} \in(a, b)\right\}-(b-a) N\right| .
$$

We note that sometimes in the literature the scaled quantity $N^{-1} D_{N}$ is called the discrepancy, but since our argument looks cleaner with the definition (2.2), we adopt it here.

For $\mathbf{x} \in \mathrm{T}_{k}, \mathbf{y} \in \mathrm{T}_{d-k}$ we consider the sequence

$$
\sum_{j=1}^{k} x_{j} \varphi_{j}(n)+\sum_{j=1}^{d-k} y_{j} \varphi_{k+j}(n), \quad n \in \mathbb{N},
$$

and for each $N$ we denote by $D_{\varphi}(\mathbf{x}, \mathbf{y} ; N)$ the corresponding discrepancy of its fractional parts.

For $\rho>0$ let

$$
\mathfrak{M}_{k, \rho}(\boldsymbol{\varphi}, N)=\int_{\mathbf{T}_{k}} \sup _{\mathbf{y} \in \mathrm{T}_{d-k}} D_{\boldsymbol{\varphi}}(\mathbf{x}, \mathbf{y} ; N)^{\rho} d \mathbf{x}
$$

Theorem 2.5. Suppose that $\varphi \in \mathbb{Z}[T]^{d}$ is such that the Wronskian $W(T ; \boldsymbol{\varphi})$ does not vanish identically. Then for any $1 \leqslant \rho \leqslant 2 s(d)+d-k$ we have

$$
\mathfrak{M}_{k, \rho}(\boldsymbol{\varphi}, N) \leqslant N^{\mu(\varphi, k) \rho+o(1)}, \quad N \rightarrow \infty,
$$

where $\mu(\boldsymbol{\varphi}, k)$ is as Theorem 2.1 .

From Theorem 2.5 we derive a bound on the mean value of discrepancy over short intervals. More precisely, for each $K \in \mathbb{Z}$ denote by $D_{d}(\mathbf{u} ; K, N)$ the discrepancy of the sequence of fractional parts

$$
\left\{u_{1} n+\ldots+u_{d} n^{d}\right\}, \quad n=K+1, \ldots, K+N .
$$

Corollary 2.6. For any real positive $1 \leqslant \rho \leqslant d^{2}+2 d-1$ we have

$$
\int_{\mathrm{T}_{d}} \sup _{K \in \mathbb{Z}} D_{d}(\mathbf{u} ; K, N)^{\rho} d \mathbf{u} \leqslant N^{\mu_{d} \rho+o(1)}, \quad N \rightarrow \infty,
$$

where $\mu_{d}$ is given by Corollary 2.2. 


\section{Preliminaries}

3.1. Packing large trigonometric polynomials in boxes. We first need a box-counting estimate from [6, Lemma 3.7].

Lemma 3.1. Let $0<\alpha<1$ and let $\varepsilon$ be sufficiently small. For each $j=1, \ldots, d$ let

$$
\zeta_{j}=1 /\left\lceil N^{e_{j}+1+\varepsilon-\alpha}\right\rceil,
$$

where $e_{j}=\operatorname{deg}\left(\varphi_{j}\right), j=1, \ldots, d$. We divide $\mathrm{T}_{d}$ into

$$
U=\left(\prod_{j=1}^{d} \zeta_{j}\right)^{-1}
$$

boxes of the form

$$
\left[n_{1} \zeta_{1},\left(n_{1}+1\right) \zeta_{1}\right) \times \ldots \times\left[n_{d} \zeta_{d},\left(n_{d}+1\right) \zeta_{d}\right)
$$

where $n_{j}=1, \ldots, 1 / \zeta_{j}$ for each $j=1, \ldots, d$. Let $\mathfrak{R}$ be the collection of these boxes, and

$$
\widetilde{\mathfrak{R}}=\left\{\mathcal{R} \in \mathfrak{R}: \exists \mathbf{u} \in \mathcal{R} \text { with }\left|T_{\mathbf{a}, \boldsymbol{\varphi}}(\mathbf{u} ; N)\right| \geqslant N^{\alpha}\right\} .
$$

Then, uniformly over $\alpha$, we have

$$
\# \widetilde{\mathfrak{R}} \leqslant U N^{s(d)(1-2 \alpha)+o(1)} .
$$

3.2. The measure of the set of large Weyl sums. We need a slightly more general version of [6, Corollary 3.8]. We recall that the result of [6, Corollary 3.8] is formulated with an $T=N^{\alpha}$ for a fixed real $\alpha$, however examining the argument one can easily see that as Lemma 3.1, it is uniform with respect to $\alpha$ and thus works for an arbitrary parameter $T \geqslant 1$. More precisely, we have:

Lemma 3.2. Let $1 \leqslant T \leqslant N$. Then

$$
\begin{aligned}
\lambda\left(\left\{\mathbf{x} \in \mathrm{T}_{k}: \exists \mathbf{y} \in \mathrm{T}_{d-k}\right.\right. & \text { with } \left.\left.\left|T_{\mathbf{a}, \boldsymbol{\varphi}}(\mathbf{x}, \mathbf{y} ; N)\right| \geqslant T\right\}\right) \\
& \leqslant N^{s(d)+\sigma_{k}(\boldsymbol{\varphi})+d-k+o(1)} T^{-2 s(d)-d+k} .
\end{aligned}
$$

The following result is similar to the result of Lemma 3.2, with the change of $\widetilde{\sigma}_{k}(\boldsymbol{\varphi})$ only. Let $\mathcal{V}$ be a $k$-dimensional subspace of $\mathbb{R}^{d}$. Also recall that $\widetilde{\sigma}_{k}(\boldsymbol{\varphi})$ is given by $(2.1)$. For the orthogonal projection map $\pi_{\mathcal{V}}$, from [6, Corollary 3.11] we have the following.

Lemma 3.3. Let $1 \leqslant T \leqslant N$. Then

$$
\begin{aligned}
\lambda\left(\left\{\mathbf{x} \in \mathcal{V}: \sup _{\mathbf{u} \in \mathrm{T}_{d} \cap \pi_{\mathcal{V}}^{-1}(\mathbf{x})}\left|T_{\mathbf{a}, \boldsymbol{\varphi}}(\mathbf{u} ; N)\right| \geqslant T\right\}\right) \\
\quad \leqslant N^{s(d)+\widetilde{\sigma}_{k}(\boldsymbol{\varphi})+d-k+o(1)} T^{-2 s(d)-d+k} .
\end{aligned}
$$


We now turn to $\vartheta$-Hölder functions $f: R^{d} \rightarrow \mathbb{R}^{k}$. Indeed, applying the similar methods to the proofs of [6, Corollaries 3.8 and 3.11] we obtain Lemma 3.4 below. Since $\vartheta$-Hölder functions do not appear in [6], for the sake of completeness, we outline the proof.

Lemma 3.4. Let $f: \mathbb{R}^{d} \rightarrow \mathbb{R}^{k}$ be a $\vartheta$-Hölder function. Then for $1 \leqslant T \leqslant N$ we have

$$
\begin{aligned}
\lambda\left(\left\{\mathbf{x} \in \mathbb{R}^{k}: \sup _{\mathbf{u} \in \mathrm{T}_{d} \cap f^{-1}(\mathbf{x})} \mid\right.\right. & \left.\left.T_{\mathbf{a}, \boldsymbol{\varphi}}(\mathbf{u} ; N) \mid \geqslant T\right\}\right) \\
& \leqslant N^{s(d)+\sigma_{0}(\boldsymbol{\varphi})+d-(\delta(\boldsymbol{\varphi})+1) \vartheta k+o(1)} T^{-2 s(d)-d+\vartheta k} .
\end{aligned}
$$

Proof. First of all suppose that $T=N^{\alpha}$ for some $0<\alpha<1$. We fix some sufficiently small $\varepsilon>0$ and define the set

$$
\mathfrak{U}=\bigcup_{\mathcal{R} \in \widetilde{\mathfrak{R}}} \mathcal{R} .
$$

For $\mathcal{A} \subseteq \mathbb{R}^{d}$ denote $f(\mathcal{A})=\{f(\mathbf{x}): \mathbf{x} \in \mathcal{A}\}$. Observe that

$$
\begin{aligned}
\left\{\mathbf{x} \in \mathbb{R}^{k}: \sup _{\mathbf{u} \in \mathrm{T}_{d} \cap f^{-1}(\mathbf{x})}\left|T_{\mathbf{a}, \varphi}(\mathbf{u} ; N)\right|\right. & \geqslant T\} \\
& \subseteq f(\mathfrak{U}) \subseteq \bigcup_{\mathcal{R} \in \widetilde{\mathfrak{R}}} f(\mathcal{R}),
\end{aligned}
$$

where $\widetilde{\mathfrak{R}}$ is as in Lemma 3.1 .

Since $f$ is $\vartheta$-Hölder, for any $\mathcal{A} \subseteq \mathbb{R}^{d}$ we obtain

$$
\lambda(f(\mathcal{A})) \ll(\operatorname{diam} \mathcal{A})^{\vartheta k},
$$

where $\operatorname{diam} \mathcal{A}=\sup \{\|\mathbf{a}-\mathbf{b}\|: \mathbf{a}, \mathbf{b} \in \mathcal{A}\}$. For each $\mathcal{R} \in \widetilde{\mathfrak{R}}$, by $(3.1)$ we have

$$
\operatorname{diam} \mathcal{R} \ll N^{\alpha-1-\delta(\varphi)} .
$$

Combining with Lemma 3.1 and the estimate (3.2), we derive

$$
\begin{aligned}
\lambda\left(\left\{\mathbf{x} \in \mathbb{R}^{k}: \sup _{\mathbf{u} \in \mathrm{T}_{d} \cap f^{-1}(\mathbf{x})} \mid\right.\right. & \left.\left.T_{\mathbf{a}, \boldsymbol{\varphi}}(\mathbf{u} ; N) \mid \geqslant T\right\}\right) \\
& \leqslant \# \widetilde{\Re}(\operatorname{diam} \mathcal{R})^{\vartheta k} \\
& \ll U N^{s(d)(1-2 \alpha)} N^{(\alpha-\delta(\varphi)-1-\varepsilon) \vartheta k}
\end{aligned}
$$

Since $\varepsilon$ is arbitrary, recalling the value of $U$, we now obtain

$$
\begin{aligned}
\lambda\left(\left\{\mathbf{x} \in \mathbb{R}^{k}:\right.\right. & \left.\left.\sup _{\mathbf{u} \in \mathbf{T}_{d} \cap f^{-1}(\mathbf{x})}\left|T_{\mathbf{a}, \boldsymbol{\varphi}}(\mathbf{u} ; N)\right| \geqslant T\right\}\right) \\
& \leqslant N^{s(d)+\sigma_{0}(\boldsymbol{\varphi})+d-(\delta(\boldsymbol{\varphi})+1) \vartheta k+o(1)} N^{-2 s(d) \alpha-d \alpha+\vartheta k \alpha .} .
\end{aligned}
$$

Recalling that $T=N^{\alpha}$, we now obtain the desired result by taking $T=N^{\alpha}$. 
3.3. Discrepancy and exponential sums. We recall the classical ErdösTurán inequality (see, for instance, [7, Theorem 1.21]).

Lemma 3.5. Let $\xi_{n}, n \in \mathbb{N}$, be a sequence in $[0,1)$. Then for the discrepancy $D_{N}$ given by (2.2) and any $G \in \mathbb{N}$, we have

$$
D_{N} \leqslant 3\left(\frac{N}{G+1}+\sum_{g=1}^{G} \frac{1}{g}\left|\sum_{n=1}^{N} \mathbf{e}\left(g \xi_{n}\right)\right|\right) \text {. }
$$

\section{Proofs of Mean Value Theorems for Exponential Sums}

4.1. Proof of Theorems 2.1, 2.3, 2.4. Theorems 2.1, 2.3 and 2.4 follows by combining Lemmas 3.2, 3.3 and 3.4, respectively, with Lemma 4.1 below.

Lemma 4.1. Let $N$ be a positive large number and $F: \mathrm{T}_{k} \rightarrow[0, N]$ be a function. Suppose that there exists positive constants $a<b$ such that for any $1 \leqslant T \leqslant N$,

$$
\lambda\left(\left\{\mathbf{x} \in \mathrm{T}_{k}: F(\mathbf{x}) \geqslant T\right\}\right) \leqslant N^{a} T^{-b} .
$$

Then for any positive $\rho \leqslant b$,

$$
\int_{T_{k}} F(\mathbf{x})^{\rho} d \mathbf{x} \ll N^{\frac{\rho a}{b}} \log N .
$$

Proof. Let $R=N^{a / b}$. Note that for $T>R$ we have a nontrivial estimate in (4.1). We partition $\mathrm{T}_{k}$ into sets $\mathcal{X}_{\ell}, \ell \in \mathbb{N}$, where

$$
\mathcal{X}_{0}=\left\{\mathbf{x} \in \mathrm{T}_{k}: F(\mathbf{x}) \leqslant R\right\}
$$

and for $\ell \geqslant 1$,

$$
\mathcal{X}_{\ell}=\left\{\mathrm{x} \in \mathrm{T}_{k}: 2^{\ell-1} R<F(\mathbf{x}) \leqslant 2^{\ell} R\right\} .
$$

By our assumption (4.1) we have

$$
\lambda\left(\mathcal{X}_{\ell}\right) \ll N^{a}\left(2^{\ell} R\right)^{-b} .
$$

Clearly for some $L=O(\log N)$ we have $\mathcal{X}_{\ell}=\emptyset$. Therefore,

$$
\begin{aligned}
\int_{\mathrm{T}_{k}} F(\mathbf{x})^{\rho} d \mathbf{x} & =\sum_{\ell=0}^{L} \int_{\mathcal{X}_{\ell}} F(\mathbf{x})^{\rho} d \mathbf{x} \\
& \ll R^{\rho}+\sum_{\ell=1}^{L}\left(2^{\ell} R\right)^{\rho} N^{a}\left(2^{\ell} R\right)^{-b} \\
& \ll R^{\rho}+N^{a} R^{\rho-b} \sum_{\ell=1}^{L} 2^{\ell(\rho-b)} .
\end{aligned}
$$


By the choice of $R=N^{a / b}$ and the condition that $\rho \leqslant b$ we obtain the desired bound.

4.2. Proof of Corollary 2.2. For $K \in \mathbb{Z}$, recall that Weyl sums over short intervals are defined as follows

$$
S_{d}(\mathbf{u} ; K, N)=\sum_{n=K+1}^{K+N} \mathbf{e}\left(u_{1} n+\ldots+u_{d} n^{d}\right) .
$$

We write

$$
S_{d}(\mathbf{u} ; K, N)=\sum_{n=1}^{N} \mathbf{e}\left(u_{1}(n+K)+\ldots+u_{d}(n+K)^{d}\right) .
$$

We observe that in the polynomial identity

$$
\begin{aligned}
u_{1}(T+K)+\ldots & +u_{d}(T+K)^{d} \\
& =v_{0}+v_{1} T+\ldots+v_{d-1} T^{d-1}+u_{d} T^{d} \in \mathbb{R}[T],
\end{aligned}
$$

where for $j=0,1, \ldots, d-1$, each $v_{j}$, depends only on $u_{1}, \ldots, u_{d}$ and $K$. It follows that

$$
\sup _{K \in \mathbb{Z}}\left|S_{d}(\mathbf{u} ; K, N)\right| \leqslant \sup _{\left(v_{1}, \ldots, v_{d-1}\right) \in \mathbf{T}_{d-1}}\left|S_{d}\left(\left(v_{1}, \ldots, v_{d-1}, u_{d}\right) ; N\right)\right| .
$$

Note that for any fixed $u_{d}$ for any $\left(u_{1}, \ldots, u_{d-1}\right) \in T_{d-1}$ the estimate 4.2 holds for $\mathbf{u}=\left(u_{1}, \ldots, u_{d}\right)$. Thus we obtain

$$
\begin{aligned}
\int_{T_{d}} \sup _{K \in \mathbb{Z}}\left|S_{d}(\mathbf{u} ; K, N)\right|^{\rho} d \mathbf{u} \\
\quad=\int_{0}^{1}\left(\int_{T_{d-1}} \sup _{K \in \mathbb{Z}}\left|S_{d}(\mathbf{u} ; K, N)\right|^{\rho} d u_{1} \ldots d u_{d-1}\right) d u_{d} \\
\leqslant \int_{0}^{1} \sup _{\left(v_{1}, \ldots, v_{d-1}\right) \in \mathrm{T}_{d-1}}\left|S_{d}\left(\left(v_{1}, \ldots, v_{d-1}, u_{d}\right) ; N\right)\right|^{\rho} d u_{d} .
\end{aligned}
$$

Hence Theorem 2.1, applied $k=1, \varphi_{1}(T)=T^{d}, \varphi_{i}(T)=T^{i-1}$ for $i=2, \ldots, d$, and thus with $\sigma_{1}(\varphi)=d(d-1) / 2$, yields the desired bound.

\section{Proofs of Mean Value Theorems for the Discrepancy}

5.1. Proof of Theorem 2.5. For any $\mathbf{x} \in \mathrm{T}_{k}, \mathbf{y} \in \mathrm{T}_{d-k}$, by Lemma 3.5 for any $G \in \mathbb{N}$ we obtain

$$
D_{\varphi}(\mathbf{x}, \mathbf{y} ; N) \ll \frac{N}{G}+\sum_{g=1}^{G} \frac{1}{g}\left|T_{\varphi}(g \mathbf{x}, g \mathbf{y} ; N)\right|,
$$


and therefore

$$
\sup _{\mathbf{y} \in \mathrm{T}_{d-k}} D_{\varphi}(\mathbf{x}, \mathbf{y} ; N) \ll \frac{N}{G}+\sum_{g=1}^{G} \frac{1}{g} \sup _{\mathbf{y} \in \mathrm{T}_{d-k}}\left|T_{\varphi}(g \mathbf{x}, \mathbf{y} ; N)\right| .
$$

We now use the following invariant property of Lebesgue measure on a torus.

Lemma 5.1. Let $F: \mathrm{T}_{k} \rightarrow[0, N]$ be a continuous function. Then for any integer $g \neq 0$ we have

$$
\int_{\mathrm{T}_{k}} F(g \mathbf{x}) d \mathbf{x}=\int_{\mathrm{T}_{k}} F(\mathbf{x}) d \mathbf{x}
$$

Proof. For any Borel set $\mathcal{A} \subseteq \mathrm{T}_{k}$ and any integer $g \neq 0$, we have (for a proof see $[11$, Section 3$])$

$$
\lambda\left(\left\{\mathbf{x} \in \mathrm{T}_{k}: g \mathbf{x} \in \mathcal{A}\right\}\right)=\lambda(\mathcal{A}),
$$

which is the same as the identity

$$
\int_{\mathrm{T}_{k}} \mathbf{1}_{\mathcal{A}}(g \mathbf{x}) d \mathbf{x}=\int_{\mathrm{T}_{k}} \mathbf{1}_{\mathcal{A}}(\mathbf{x}) d \mathbf{x}
$$

where $\mathbf{1}_{\mathcal{A}}$ is the characteristic function of $\mathcal{A}$. Thus (5.2) holds when $F=\mathbf{1}_{\mathcal{A}}$. It follows that the identity (5.2) still holds when $F$ is a finite linear combination of characteristic functions, that is

$$
F(\mathbf{x})=\sum_{j=1}^{J} a_{j} \mathbf{1}_{\mathcal{A}_{j}}(\mathbf{x})
$$

for sets $\mathcal{A}_{j} \subseteq \mathrm{T}_{k}, j=1, \ldots, J$. Since any continuous function can be arbitrary approximated by a finite linear combination of such functions, the desired identity follows.

Here $\|f(\mathbf{x})\|_{\rho}$ denote the $L^{\rho}\left(\mathrm{T}_{k}\right)$-norm of a function $f$ on $\mathrm{T}_{k}$. Then by (5.1) and the Minkowski inequality,

$$
\left\|\sup _{\mathbf{y} \in \mathbf{T}_{d-k}} D_{\boldsymbol{\varphi}}(\mathbf{x}, \mathbf{y} ; N)\right\|_{\rho} \ll \frac{N}{G}+\sum_{g=1}^{G} \frac{1}{g}\left\|\sup _{\mathbf{y} \in \mathbf{T}_{d-k}}\left|T_{\boldsymbol{\varphi}}(g \mathbf{x}, \mathbf{y} ; N)\right|\right\|_{\rho} .
$$

For any positive integer $g \neq 0$, Lemma 5.1 implies

$$
\left\|\sup _{\mathbf{y} \in \mathrm{T}_{d-k}}\left|T_{\boldsymbol{\varphi}}(g \mathbf{x}, \mathbf{y} ; N)\right|\right\|_{\rho}=\left\|\sup _{\mathbf{y} \in \mathrm{T}_{d-k}}\left|T_{\boldsymbol{\varphi}}(\mathbf{x}, \mathbf{y} ; N)\right|\right\|_{\rho},
$$


which together with (5.3) and Theorem 2.1 yileds

$$
\begin{aligned}
\left\|\sup _{\mathbf{y} \in \mathrm{T}_{d-k}} D_{\varphi}(\mathbf{x}, \mathbf{y} ; N)\right\|_{\rho} & \ll \frac{N}{G}+\left\|\sup _{\mathbf{y} \in \mathrm{T}_{d-k}}\left|T_{\varphi}(\mathbf{x}, \mathbf{y} ; N)\right|\right\|_{\rho} \log G \\
& \ll \frac{N}{G}+N^{\mu(\boldsymbol{\varphi}, k)+o(1)} \log G .
\end{aligned}
$$

Choosing $G=N^{1-\mu(\varphi, k)}$, we derive the desired result.

5.2. Proof of Corollary 2.6. Recall that $D_{d}(\mathbf{u} ; K, N)$ is the discrepancy of the sequence of fractional parts

$$
\left\{u_{1} n+\ldots+u_{d} n^{d}\right\}, \quad n=K+1, \ldots, K+N .
$$

Clearly this sequence is same as

$$
\left\{u_{1}(n+K)+\ldots+u_{d}(n+K)^{d}\right\}, \quad n=1, \ldots, N,
$$

and thus as before, see (4.2), we see that this sequence is the same as

$$
\left\{v_{0}+v_{1} n+\ldots+v_{d-1} n^{d-1}+u_{d} n^{d}\right\}, \quad n=1, \ldots, N,
$$

where for $j=0,1, \ldots, d-1$, each $v_{j}$, depends only on $u_{1}, \ldots, u_{d}$ and $K$. Furthermore let $\mathbf{u}^{*}=\left(v_{1}, \ldots, v_{d-1}, u_{d}\right)$. It is not hard to see that the influence of the discarded constant term $v_{0}$ can be absorbed in a constant factor and does not change the order of magnitude of the discrepancy. More, precisely, we have

$$
D_{d}\left(\mathbf{u}^{*} ; N\right) \ll D_{d}(\mathbf{u} ; K, N) \ll D_{d}\left(\mathbf{u}^{*} ; N\right),
$$

where the implied constant is absolute. It follows that

$$
\sup _{K \in \mathbb{Z}} D_{d}(\mathbf{u} ; K, N) \ll \sup _{\left(v_{1}, \ldots, v_{d-1}\right) \in \mathrm{T}_{d-1}} D_{d}\left(\left(v_{1}, \ldots, v_{d-1}, u_{d}\right), N\right) .
$$

Using the similar arguments as in the proof of Corollary 2.2 and applying Theorem 2.5, with $k=1, \varphi_{1}(T)=T^{d}, \varphi_{i}(T)=T^{i-1}$ for $i=2, \ldots, d$, and thus with $\sigma_{1}(\boldsymbol{\varphi})=d(d-1) / 2$, we obtain the desired bound.

\section{Comments and Open Questions}

6.1. Special cases of $d=2$ and $d=3$. We show that for special cases of $d=2$ and $d=3$ for the moments

$$
\begin{aligned}
& \mathcal{M}_{2,2}(N)=\int_{0}^{1} \sup _{y \in[0,1]}\left|\sum_{n=1}^{N} \mathbf{e}\left(x n^{2}+y n\right)\right|^{2} d x \\
& \mathcal{M}_{3,4}(N)=\int_{0}^{1} \sup _{y, z \in[0,1]}\left|\sum_{n=1}^{N} \mathbf{e}\left(x n^{3}+y n^{2}+z n\right)\right|^{4} d x
\end{aligned}
$$


we have better bounds (nearly optimal) than the bounds in Theorem 2.1.

Applying Theorem 2.1 with $d=2, k=1, \sigma_{k}(\boldsymbol{\varphi})=1, \rho=2$ and with $d=3, k=1, \sigma_{k}(\boldsymbol{\varphi})=3, \rho=4$ we derive that

$$
\mathcal{M}_{2,2}(N) \leqslant N^{10 / 7+o(1)} \quad \text { and } \quad \mathcal{M}_{3,4}(N) \leqslant N^{22 / 7+o(1)},
$$

respectively. On the other hand, we have the lower bounds

$$
\mathcal{M}_{2,2}(N) \geqslant \int_{0}^{1} \int_{0}^{1}\left|\sum_{n=1}^{N} \mathbf{e}\left(x n^{2}+y n\right)\right|^{2} d y d x=N,
$$

and

$$
\begin{aligned}
\mathcal{M}_{3,4}(N) & \geqslant \int_{0}^{1} \int_{0}^{1} \int_{0}^{1}\left|\sum_{n=1}^{N} \mathbf{e}\left(x n^{3}+y n^{2}+z n\right)\right|^{4} d z d y d x \\
& \geqslant 2 N^{2}+O(N),
\end{aligned}
$$

see, for example, [10, Section 1].

Now we use a different way to bound the square mean values (6.1) and reduce the gap between the above lower and upper bounds.

For $d=2$ we need the following well known inequality, for a proof see $[8$, Inequality (8.8)]:

$$
\sup _{y \in[0,1]}\left|\sum_{n=1}^{N} \mathbf{e}\left(x n^{2}+y n\right)\right|^{2} \ll N+\sum_{h=1}^{N} \min \left\{\frac{1}{\|2 h x\|}, N\right\},
$$

where $\|x\|=\min \{|x-n|: n \in \mathbb{Z}\}$ denotes the distance of $x$ to the nearest integer. Moreover, for any positive integer $h$, applying Lemma 5.1 we obtain that uniformly over $h$, we have

$$
\int_{0}^{1} \min \left\{\frac{1}{\|2 h x\|}, N\right\} d x=\int_{0}^{1} \min \left\{\frac{1}{\|x\|}, N\right\} d x \ll \log N,
$$

hence

$$
\mathcal{M}_{2,2}(N) \ll N \log N
$$

Note that this is tight except, possibly, for the logarithm factor.

For $d=3$ we use that by [8, Proposition 8.2]:

$$
\begin{aligned}
\sup _{y, z \in[0,1]}\left|\sum_{n=1}^{N} \mathbf{e}\left(x n^{3}+y n^{2}+z n\right)\right| & \ll N \sum_{g=-N}^{N} \sum_{h=-N}^{N} \min \left\{\frac{1}{\|6 g h x\|}, N\right\} \\
& \ll N^{3}+N \sum_{\substack{g, h=-N \\
g h \neq 0}}^{N} \min \left\{\frac{1}{\|6 g h x\|}, N\right\},
\end{aligned}
$$


and arguing as before we obtain

$$
\mathcal{M}_{3,4}(N) \ll N^{3} \log N
$$

It is natural to ask whether similar arguments can improve Theorem 2.1 for higher degrees. Note that typically methods based on the Vinogradov mean value theorem yield better bounds for higher degrees. Indeed this also happens here, which means that Theorem 2.1 gives stronger bounds than using [8, Proposition 8.2] for $d \geqslant 4$ and applying the above arguments.

6.2. Open questions. It is interesting to try to use the "self-improving" idea of [6] and in particular [6, Corollary 3.9] to obtain stronger results in the case when one of the polynomials $\varphi_{k+1} \ldots, \varphi_{d}$ is linear. There are however some obstacles which the authors have not been able to overcome.

It is also natural to study the mixed mean values

$$
M_{k, \rho, \tau}(\mathbf{a}, \boldsymbol{\varphi}, N)=\int_{\mathbf{x} \in[0,1]^{k}}\left(\int_{\mathbf{y} \in[0,1]^{d-k}}\left|T_{\mathbf{a}, \boldsymbol{\varphi}}(\mathbf{x}, \mathbf{y} ; N)\right|^{\tau} d \mathbf{y}\right)^{\rho} d \mathbf{x},
$$

and obtain bounds which are stronger than those following from the trivial inequality

$$
M_{k, \rho, \tau}(\mathbf{a}, \boldsymbol{\varphi}, N) \leqslant M_{k, \rho \tau}(\mathbf{a}, \boldsymbol{\varphi}, N)
$$

and Theorem 2.1. The case of the classical Weyl sums is of special interest.

\section{ACKNOWLEDGEMENT}

This work was supported by ARC Grant DP170100786.

\section{REFERENCES}

[1] R. C. Baker, 'Metric number theory and the large sieve', J. London Math. Soc., 24 (1981), 34-40. 1

[2] J. Bourgain, 'On the Vinogradov mean value', Proc. Steklov Math. Inst., 296 (2017), 30-40. 2

[3] J. Bourgain, C. Demeter and L. Guth, 'Proof of the main conjecture in Vinogradov's mean value theorem for degrees higher than three', Ann. Math., 184 (2016), 633-682. 2

[4] J. Brüdern, 'Approximations to Weyl sums', Acta Arith., 184 (2018), 287-296. 2

[5] J. Brüdern and D. Daemen, 'Imperfect mimesis of Weyl sums', Internat. Math. Res. Notices,, 2009 (2009), 3112-3126. 2

[6] C. Chen and I. E. Shparlinski, 'New bounds of Weyl sums', Intern. Math. Res. Notices, (to appear). 3, 7, 8, 9, 15

[7] M. Drmota and R. Tichy, Sequences, discrepancies and applications, SpringerVerlag, Berlin, 1997. 10 
[8] H. Iwaniec and E. Kowalski, Analytic number theory, Amer. Math. Soc., Providence, RI, 2004. 2, 14, 15

[9] H. Weyl, 'Über die Gleichverteilung von Zahlen mod Eins', Math. Ann., 77 (1916), 313-352. 1

[10] T. D. Wooley, 'The cubic case of the main conjecture in Vinogradov's mean value theorem', Adv. in Math., 294 (2016), 532-561. 2, 14

[11] T. D. Wooley, 'Perturbations of Weyl sums', Internat. Math. Res. Notices, 2016 (2016), 2632-2646. 2, 3, 7, 12

[12] T. D. Wooley, 'Nested efficient congruencing and relatives of Vinogradov's mean value theorem', Proc. London Math. Soc., 118 (2019), 942-1016. 2

Department of Pure Mathematics, University of New South Wales, Sydney, NSW 2052, Australia

E-mail address: changhao.chenm@gmail.com

Department of Pure Mathematics, University of New South Wales, Sydney, NSW 2052, Australia

E-mail address: igor.shparlinski@unsw.edu.au 